\title{
Impact of infant 13-valent pneumococcal conjugate vaccine on serotypes in adult pneumonia
}

\author{
Chamira Rodrigo ${ }^{1}$, Thomas Bewick$^{1}$, Carmen Sheppard ${ }^{2}$, Sonia Greenwood ${ }^{1}$, \\ Tricia M. Mckeever ${ }^{3}$, Caroline L. Trotter ${ }^{4}$, Mary Slack², Robert George ${ }^{2}$ and \\ Wei Shen $\operatorname{Lim}^{1}$
}

Affiliations: 'Dept of Respiratory Medicine, City Hospital Campus, Nottingham University Hospitals NHS Trust, Nottingham, UK. ${ }^{2}$ Respiratory and Vaccine Preventable Bacteria Reference Unit, Public Health England, Microbiology Services Division, London, UK. ${ }^{3}$ Division of Epidemiology and Public Health, University of Nottingham, Nottingham, UK. ${ }^{4}$ Disease Dynamics Unit, Dept of Veterinary Medicine, University of Cambridge, Cambridge, UK.

Correspondence: C. Rodrigo, Dept of Respiratory Medicine, David Evans Building, City Hospital Campus, Nottingham University Hospitals NHS Trust, Hucknall Road, Nottingham. NG51PB, UK.

E-mail: chamiraddoctors.org.uk

ABSTRACT Infant 13-valent pneumococcal conjugate vaccination (PCV13) was introduced to the UK in 2010. Its impact on serotypes implicated in adult non-bacteraemic pneumococcal pneumonia is not known.

Beginning in 2008, a 5-year prospective cohort study of adults admitted to hospital with communityacquired pneumonia (CAP) was conducted. Pneumococcal serotype was established using a validated multiplex immunoassay (Bio-Plex; Bio-Rad, Hercules, CA, USA).

The overall incidence for hospitalised CAP and pneumococcal CAP was 79.9 (95\% CI 76.6-83.3) and 23.4 (95\% CI 21.6-25.3) per 100000 population, respectively. A decline in CAP (incidence rate ratio (IRR) per year $0.96,95 \%$ CI $0.94-0.99 ; \mathrm{p}=0.016$ ) and pneumococcal CAP (IRR per year $0.84,95 \%$ CI $0.80-0.89$; $\mathrm{p}<0.001)$ was observed over the 5 -year period of the study. Between the pre- and post-PCV13 periods of the study, the incidence of CAP due to serotypes included in the PCV7 declined by $88 \%$ (IRR 0.12, 95\% CI 0.08-0.20; $\mathrm{p}<0.001$ ), and CAP due to the additional 6 serotypes in PCV13 declined by 30\% (IRR 0.70 , 95\% CI 0.51-0.96; $\mathrm{p}=0.024)$.

Incidence of adult pneumococcal pneumonia declined over the last 5 years, with serotypes included in PCV13 declining post-PCV13 introduction, indicating early herd protection effects from PCV13 infant vaccination on adult non-bacteraemic disease. These effects may accrue over the coming years with implications for national pneumococcal vaccination policies in adults.

@ERSpublications

This is the first study to indicate herd protection from infant PCV13 on adult non-bacteraemic pneumococcal pneumonia http://ow.ly/HHP75

Editorial comment in Eur Respir J 2015; 45: 1524-1525 [DOI: 10.1183/09031936.00064015].

This article has supplementary material available from erj.ersjournals.com

Received: Oct 042014 | Accepted after revision: Dec 162014 | First published online: March 182015

Support statement: This report is independent research arising from a Biomedical Research Fellowship supported by the National Institute for Health Research (NIHR), and an unrestricted investigator-initiated research grant from Wyeth (previously) and Pfizer. The study concept was developed and agreed by the authors with no input from the funding bodies. Pfizer (previously Wyeth), had no part in the design or execution of the study, the analysis and interpretation of the results, the writing of this manuscript or the decision to submit for publication. The data are the sole responsibility of the authors and the sponsor for the study was Nottingham University Hospitals NHS Trust. The views expressed in this publication are those of the authors and not necessarily those of the National Health Service, the National for Institute for Health Research or Public Health England. Funding information for this article has been deposited with FundRef.

Conflict of interest: Disclosures can be found alongside the online version of this article at erj.ersjournals.com

Copyright OERS 2015 


\section{Introduction}

Community-acquired pneumonia (CAP) is the leading cause of sepsis presenting to hospitals, and in the UK, results in over 100000 admissions per year $[1,2]$. The associated mortality is $14-25 \%$, with older age groups particularly at risk $[2,3]$. Streptococcus pneumonia is the pathogen implicated in $37-48 \%$ of CAP $[3,4]$. A 7 -valent pneumococcal conjugate vaccine (PCV7) was introduced into the infant immunisation programme in the UK in September 2006, followed by a PCV13 in April 2010. For adults in the UK, only the 23-valent pneumococcal polysaccharide vaccine (PPV23) has been recommended for routine immunisation, except in severely immunocompromised adults where the recommendation is for a single dose of PCV13 followed by PPV23 [5].

Following the introduction of PCVs, a reduction in invasive pneumococcal disease (IPD) and IPD due to vaccine-type serotypes has been reported among children in several countries including the UK, with varying degrees of serotype replacement with nonvaccine-type serotypes $[6,7]$. Reductions in the incidence of PCV7-type IPD were also seen in the unvaccinated adult population, due to herd protection effects. However, invasive pneumococcal disease accounts for only $7-14 \%$ of pneumococcal CAP in adults presenting to hospitals $[3,8]$. Changes in pneumococcal serotype distribution implicated in adult non-bacteraemic pneumococcal disease as a consequence of the introduction of PCV13 have not been reported to date.

A key economic evaluation from 2012, on the cost-effectiveness of vaccinating at risk groups aged $\geqslant 2$ years with PCV13, reported that the two most influential variables on outcome were the assumed herd protection effects of infant pneumococcal vaccination and the direct vaccine effectiveness against non-bacteraemic pneumococcal disease [9]. This study addresses the first of these factors.

We report the changes in pneumococcal serotypes in the years prior to and following PCV13 introduction in the UK from a 5-year prospective cohort study of adults hospitalised with predominantly non-bacteraemic CAP.

\section{Method}

Beginning in September 2008, a prospective cohort study of adults with CAP admitted to two large teaching hospitals in Nottingham, UK, was conducted over 5 years. Adults (patients aged $\geqslant 16$ years) with symptoms suggestive of lower respiratory tract infection (at least one of breathlessness, cough, sputum or fever), with new infiltrates on chest radiography consistent with pneumonia, and treated by the admitting clinical team for CAP, were included in this study. Exclusion criteria were post-obstruction pneumonia due to lung cancer, active tuberculosis, discharged from hospital within the preceding 10 days, and adults with aspiration pneumonia. Study investigators attended acute admission units, hospital wards and critical care units on a daily basis to identify adults with CAP. Following informed consent, a standardised proforma was used to collect information regarding patient demographics, clinical information, microbiological investigations, radiological findings and outcome measures. If an adult was unable to consent to the study, due to acute confusion or cognitive impairment, informed assent was obtained from a relative. All adults were managed at the discretion of the admitting clinical team. Ethics approval was granted by the Nottingham Regional Ethics Committee (Nottingham, UK).

Urine samples were collected from participants following admission and sent to the Nottingham University Hospitals' Department of Clinical Microbiology, a regional Public Health England laboratory. Samples were tested using the BinaxNOW (Alere, Stockport, UK) immunochromatographic test kit according to manufacturer's instructions and placed in frozen storage. The sensitivity and specificity of the BinaxNOW test in a recent meta-analysis was $74 \%$ and $97 \%$, respectively [10]. Samples were subsequently transported, in batches, to the Respiratory and Vaccine Preventable Bacteria Reference Unit at the Public Health England, Colindale (UK). Pneumococcal serotyping was carried out using a validated multiplex immunoassay (Bio-Plex; Bio-Rad, Hercules, CA, USA) capable of detecting 14 pneumococcal serotypes/ serogroups $(1,3,4,5,6 \mathrm{~A} / \mathrm{C}, 6 \mathrm{~B}, 7 \mathrm{~F} / \mathrm{A}, 8,9 \mathrm{~V}, 14,18,19 \mathrm{~A}, 19 \mathrm{~F}$ and $23 \mathrm{~F})$ with a sensitivity and specificity of 79 and 99\%, respectively [11]. The Bio-Plex assay does not differentiate between serotypes $6 \mathrm{~A}$ versus $6 \mathrm{C}, 7 \mathrm{~F}$ versus $7 \mathrm{~A}$, and the individual serotypes within serogroup 18 (18F, 18A, 18B and 18C). In bacteraemic adults, serotype was also determined using slide agglutination using the full set of serotyping latex and factor serum available from Statens Serum Institut (Copenhagen, Denmark). This method is capable of determining all serotypes of pneumococci except the newly described serotype 11E. All included adults $(n=2229)$ were tested using BinaxNOW and Bio-Plex, and $2106(94.5 \%)$ had blood cultures performed. Adults with a positive BinaxNOW test, Bio-Plex test or blood culture for S. pneumoniae were deemed to have pneumococcal CAP. Respiratory samples were not used for the diagnosis of pneumococcal disease or determination of pneumococcal serotype in this study, due to the poor sensitivity and specificity reported in previous studies $[12,13]$. 
Pneumococcal serotypes were categorised as follows: 4, 6B, 9 V, 14, 18C, 19F and 23F as "PCV7 serotypes"; 1, 3, 5, 6A/C, 7F/A and 19F as "additional PCV13 serotypes", and the remaining detected serotypes (serotype 8 from Bio-Plex and serotypes obtained from bacteraemic adults that are not amongst the detectable Bio-Plex serotypes) as "other serotypes". Individuals with CAP who were BinaxNOW positive, but were Bio-Plex negative and blood culture negative for Streptococcus pneumonia, were deemed to have "untyped" pneumococcal CAP.

Annual incidence rates were derived using data from the Office of National Statistics for the Greater Nottingham area, which also provided local population data stratified according to age groups (16-24, 25$44,45-64,65-74,75-84$ and $>85$ years) [14]. In the first analysis, trends in the overall incidence of CAP, pneumococcal CAP and serotype categories during the study period were examined (from September 2008 to August 2013). For the second analysis comparing periods before and after PCV13 introduction, incidence rates in the first year of the study (from September 2008 to August 2009) were compared with the overall incidence rate for years 3-5 combined (from September 2010 to August 2013). As PCV13 was introduced in April 2010 in the UK, the second year of the study (from September 2009 to August 2010) was considered to be the "transitional period" for the change from PCV7 to PCV13.

Statistical analyses were performed in STATA (version 12; StataCorp, College Station, TX, USA). Poisson regression models were used to assess year to year variation in incidence, for linear trend analysis, and comparison of incidence between pre- and post-PCV13 periods, together with $95 \%$ confidence intervals.

\section{Results}

From the 2702 eligible adults identified over the 5-year period, 2321 consented to the study. Of these, 16 were excluded due to an alternative final diagnosis and 76 were excluded as they were unable to provide a urine sample, leaving 2229 in the cohort for analyses. The median age of the cohort was 71.0 years (interquartile range (IQR) 55.1-80.5) and 992 (44.5\%) were female (table 1). Pneumonia severity was low, moderate and high in 1042 (46.8\%), 673 (30.2\%) and 514 (23.1\%) adults, respectively; 267 (12.0\%) adults

TABLE 1 Baseline characteristics, disease severity and outcomes of community-acquired pneumonia (CAP) cohort and pneumococcal cohort

CAP cohort

\section{Patients}

Demographics

Age years

Male

Nursing home residence

WHO performance status $\geqslant 2$

Comorbid illness

Charlson Comorbidity Index

Malignancy

Liver disease

Congestive cardiac failure

Ischaemic heart disease

Chronic kidney disease

Asthma

COPD

Diabetes

Stroke

Dementia

HIV

Disease severity

CURB-65 0-1

CURB-65 2

CURB-65 3-5

Outcomes

ICU admission

30-day in-patient mortality
2229

$71.0(55.1-80.5)$
$1237(55.5)$
$93(4.2)$
$346(15.5)$

$4.0(3.0-6.0)$
$170(7.6)$
$22(1.0)$
$144(6.5)$
$396(17.8)$
$155(7.0)$
$265(11.9)$
$475(21.3)$
$307(13.8)$
$201(9.0)$
$47(2.1)$
$11(0.5)$
$1042(46.8)$
$673(30.2)$
$514(23.1)$
$267(12.0)$
$183(8.2)$

Pneumococcal cohort

653

$69.2(50.2-79.8)$

$326(49.9)$

$30(4.6)$

$57(16.0)$

$4.0(2.0-6.0)$

$40(6.1)$

$10(1.5)$

36 (5.5)

$112(17.2)$

$48(7.4)$

$85(13.0)$

$117(17.9)$

84 (12.9)

68 (10.4)

21 (3.2)

$4(0.6)$

272 (41.7)

199 (30.5)

182 (27.9)

95 (14.5)

$41(6.3)$

Data are presented as $\mathrm{n}$, median (interquartile range) or $\mathrm{n} n$ (\%). WHO: World Health Organization; COPD: chronic obstructive pulmonary disease; CURB-65: confusion, urea $>7 \mathrm{mmol} \cdot \mathrm{L}^{-1}$, respiratory rate $>30$ breaths $\mathrm{min}^{-1}$, blood pressure $<90 \mathrm{mmHg}$ (systolic) $\leqslant 60 \mathrm{mmHg}$ (diastolic), age $\geqslant 65$ years; ICU: intensive care unit. 
were admitted to a critical care unit and 30-day mortality was $8.2 \%(n=183)$. Disease severity according to study year is shown in table S1. The percentages of children that had received two doses of PCV by their first birthday in the Nottingham City Primary Care Trust area were 92.1\%, 94.2\%, 95.0\%, 95.8\%, 95.5\% and $96.2 \%$ for the years $2008,2009,2010,2011,2012$ and 2013, respectively [15]. The vaccine uptake rates of the PPV23 in adults aged $\geqslant 65$ years in the UK were $69.0 \%, 68.2 \%, 70.5 \%, 68.3 \%$ and $69.1 \%$ for the years 2008-2009, 2009-2010, 2010-2011, 2011-2012 and 2012-2013, respectively [15].

Pneumococcal aetiology was identified in 653 (29.3\%) adults (table 1). Of these, 407 (62.3\%), 411 (62.9\%) and $87(13.3 \%)$ had positive BinaxNOW test, Bio-Plex assay and blood culture result, respectively, (fig. S1). A serotype or serogroup was determined in 444 (68.0\%) out of 653 adults with pneumococcal aetiology; eight individuals had two serotypes/serogroups identified simultaneously $(15 \mathrm{~A}+18(\mathrm{n}=1), 1+22 \mathrm{~F}$ $(n=1), 4+1(n=2), 4+8(n=3)$ and $6 B+9 V(n=1))$ and were excluded from the analyses specific to serotype trends. From the 1368 adults who were aged $\geqslant 65$ years, $753(55.0 \%)$ had received PPV23 and from the 383 adults aged $\geqslant 65$ years with pneumococcal pneumonia 213 (55.6\%) had received PPV23.

\section{Trends in hospitalised CAP and pneumococcal pneumonia}

The overall incidence of CAP for the duration of the study was 79.9 (95\% confidence interval 76.6-83.3) per 100000 population (table 2). A decline in CAP incidence was observed over the 5 years (incidence rate ratio (IRR) per year $0.96,95 \%$ CI $0.94-0.99 ; \mathrm{p}=0.016$ ) (table 3 ). The largest absolute and relative reduction in CAP incidence was seen in adults aged $\geqslant 85$ years (rate difference between 2008-2009 and 2012-2013, 625.0 cases per 100000 population; relative reduction 59.4\%). Incidence rates for CAP increased with increased age groups across all years (IRR per increasing age group 2.25, 95\% CI 2.19-2.32; p<0.001).

The overall incidence for pneumococcal CAP was 23.4 (95\% CI 21.6-25.3) per 100000 population over the 5 -year period. A decline in pneumococcal CAP incidence rate was observed over the 5 years (IRR per year $0.84,95 \%$ CI $0.80-0.89 ; \mathrm{p}<0.001$ ), with the largest absolute and relative reduction seen in the eldest age group (rate difference between 2008-2009 and 2012-2013, 250 cases per 100000 population, relative reduction 60.0\%) (tables 2 and 3, and fig. S2). Older age groups consistently had higher incidence rates of pneumococcal CAP throughout the 5 years (IRR per increasing age group 2.17, 95\% CI 2.05-2.29; $<<0.001$ ).

\section{Trends in pneumococcal serotypes}

Of 436 adults with a single pneumococcal serotype identified, 94 (21.6\%), 247 (56.7\%) and 95 (21.8\%) had CAP due to PCV7, additional PCV13, and other serotypes, respectively. The overall incidence rates of CAP due to PCV7 serotypes, additional PCV13 serotypes, and other serotypes were 3.4 (95\% CI 2.7-4.1), 8.9 (95\% CI 7.8-10.0) and 3.4 (95\%CI 2.8-4.2) per 100000 population, respectively. The incidence of CAP due to PCV7 serotypes was highest in 2008-09 (11.1 (95\% CI 8.5-14.2) cases per 100000 population), after which rates declined to 1.6 (95\% CI 0.7-3.1) cases per 100000 population in 2009-2010, and remained low throughout the remainder of the study (table 2 and fig. 1). The 5-year linear trend in CAP incidence due to PCV7 serotypes showed a marked decline (IRR per year 0.52 , 95\% CI 0.43-0.62; $\mathrm{p}<0.001$ ) (table 3). The predominant PCV7 serotypes identified were serotypes 4 and 14, accounting for $70.2 \%$ of all PCV7 serotypes (fig. 2). Reductions in CAP due to serotypes 4 and 14 were observed over the 5 -year period (IRR per year 0.41 (95\%CI 0.24-0.69) and 0.28 (95\% CI 0.19-0.41) for serotypes 4 and 14, respectively). The remaining PCV7 serotypes (6B, $9 \mathrm{~V} \mathrm{18C}, 19 \mathrm{~F}$ and $23 \mathrm{~F}$ ) were much less commonly implicated ( $<10$ cases each over the study period).

Pneumonia due to additional PCV13 serotypes peaked during 2009-2010 (11.5 (95\% CI 8.8-14.6) cases per 100000 population), but gradually declined over the subsequent 3 years (8.1 (95\% CI 5.9-10.8), 7.9 (95\% CI 5.7-10.6) and 6.3 (95\% CI 4.4-8.7) cases per 100000 population for years $2010-11,2011-12$ and 2012-13, respectively) (table 2 and fig. 2). The CAP incidence rate due to additional PCV13 serotypes declined between years one and five (IRR per year $0.87,95 \%$ CI $0.80-0.95, p=0.002$ ) (table 3). CAP due to serotypes 3 and 5 peaked during 2009-2010, and declined thereafter (fig. 2). 5-year linear trends showed a decline in CAP due to serotypes 1,3 and 5 (IRR per year $0.68,95 \%$ CI $0.56-0.82, \mathrm{p}<0.001 ; 0.58,95 \%$ CI $0.42-0.82, \mathrm{p}=0.002$; and $0.60,95 \%$ CI $0.43-0.84, \mathrm{p}=0.003$ for serotypes 1,3 and 5 , respectively). However, CAP due to serotype 7F/A increased over the 5 years (IRR per year $1.37,95 \%$ CI 1.15-1.63; p=0.001), reaching a peak in 2012-2013 with an incidence of 4.5 (95\% CI 2.9-6.6) cases per 100000 population. The other serotypes implicated in adults admitted with CAP by study year are shown in table S2.

\section{Comparison between pre- and post-PCV13 vaccination periods}

The incidence rates for CAP and pneumococcal CAP in the post-PCV13 period were lower in comparison to the pre-PCV13 period (IRR 0.85 (95\% CI 0.76-0.94) and 0.52 (95\% CI 0.44-0.62) for CAP and pneumococcal CAP, respectively) (table 4). Between the two periods CAP, due to PCV7 serotypes and additional PCV13 serotypes, also declined, although the magnitude of reduction was greater for CAP 
TABLE 2 Incidence rates of community-acquired pneumonia (CAP), pneumococcal CAP, and CAP due to 7-valent pneumococcal conjugate vaccination (PCV7), additional PCV13 and other serotypes by study year

\begin{tabular}{|c|c|c|c|c|c|c|c|c|c|c|c|}
\hline & \multirow{2}{*}{$\begin{array}{l}\text { Patient } \\
\text { age years }\end{array}$} & \multicolumn{2}{|r|}{ Year 1, 2008-2009 } & \multicolumn{2}{|r|}{ Year 2, 2009-2010 } & \multicolumn{2}{|r|}{ Year 3, 2010-2011 } & \multicolumn{2}{|r|}{ Year 4, 2011-2012 } & \multicolumn{2}{|r|}{ Year 5, 2012-2013 } \\
\hline & & $\mathrm{n}$ & Incidence $^{\#}(95 \% \mathrm{CI})$ & $\mathrm{n}$ & Incidence $^{\#}(95 \% \mathrm{CI})$ & $\mathrm{n}$ & Incidence $^{\#}(95 \% \mathrm{CI})$ & $n$ & Incidence $^{\#}(95 \% \mathrm{Cl})$ & $\mathrm{n}$ & Incidence ${ }^{\#}(95 \% \mathrm{CI})$ \\
\hline \multirow[t]{7}{*}{ CAP } & & 506 & $90.7(83.0-99.0)$ & 439 & $78.7(71.5-86.4)$ & 359 & 64.4 (57.9-71.4) & 560 & $100.4(92.3-109.1)$ & 365 & 65.4 (58.9-72.5) \\
\hline & $16-24$ & 16 & $14.0(8.0-22.7)$ & 6 & $5.3(1.9-11.4)$ & 11 & $9.6(4.8-17.2)$ & 23 & $20.1(12.8-30.2)$ & 15 & $13.1(7.4-21.6)$ \\
\hline & $25-44$ & 50 & $27.9(20.7-36.7)$ & 60 & $33.4(25.5-43.0)$ & 51 & $28.4(21.2-37.4)$ & 61 & $34.0(26.0-43.7)$ & 49 & $27.3(20.2-36.1)$ \\
\hline & $45-64$ & 97 & $59.7(48.4-72.8)$ & 91 & $56.0(45.1-68.7)$ & 82 & $50.4(40.1-62.6)$ & 131 & $80.6(67.4-95.6)$ & 95 & $58.4(47.3-71.4)$ \\
\hline & $65-74$ & 113 & $201.4(166.0-242.2)$ & 89 & 158.7 (127.4-195.2) & 77 & $137.2(108.3-171.6)$ & 107 & $190.7(156.3-230.5)$ & 77 & $137.2(108.3-171.6)$ \\
\hline & $75-84$ & 129 & $360.3(300.8-428.1)$ & 111 & $310.1(255.1-373.4)$ & 91 & $254.2(204.7-312.1)$ & 148 & $413.4(349.5-485.6)$ & 88 & $245.8(197.2-302.8)$ \\
\hline & $>85$ & 101 & $1052.1(856.9-1278.4)$ & 82 & $854.2(679.3-1060.2)$ & 47 & $489.6(359.7-651.0)$ & 90 & 937.5 (753.9-1152.3) & 41 & $427.1(306.5-579.4)$ \\
\hline \multirow[t]{7}{*}{ Pneumococcal CAP } & & 196 & $35.1(30.4-40.4)$ & 150 & $26.9(22.8-31.6)$ & 100 & $17.9(14.6-21.8)$ & 92 & $16.5(13.3-20.2)$ & 115 & $20.6(17.0-24.8)$ \\
\hline & $16-24$ & 6 & $5.2(1.9-11.4)$ & 4 & $3.5(1.0-9.0)$ & 3 & $2.6(0.5-7.7)$ & 3 & $2.6(0.5-7.7)$ & 6 & $5.2(1.9-11.4)$ \\
\hline & $25-44$ & 22 & $12.3(7.7-18.6)$ & 26 & $14.5(9.5-21.2)$ & 22 & $12.3(7.7-18.6)$ & 10 & $5.6(2.7-10.2)$ & 19 & $10.6(6.4-16.5)$ \\
\hline & $45-64$ & 40 & $24.6(17.6-33.5)$ & 31 & $19.1(13.0-27.1)$ & 24 & $14.8(9.5-22.0)$ & 20 & $12.3(7.5-19.0)$ & 26 & $16.0(10.4-23.4)$ \\
\hline & $65-74$ & 39 & $69.5(49.4-95.0)$ & 28 & $49.9(33.2-72.1)$ & 22 & $39.2(24.6-59.4)$ & 20 & $35.6(21.8-55.1)$ & 23 & $41.0(26.0-61.5)$ \\
\hline & $75-84$ & 49 & $136.9(101.3-181.0)$ & 34 & $95.0(65.8-132.7)$ & 18 & $50.3(29.8-79.5)$ & 24 & $67.0(43.0-99.8)$ & 25 & $69.8(45.2-103.1)$ \\
\hline & $>85$ & 40 & $416.7(297.7-567.4)$ & 27 & $281.2(185.3-409.2)$ & 11 & 114.6 (57.2-205.0) & 15 & $156.2(87.4-257.7)$ & 16 & 166.7 (95.3-270.7) \\
\hline \multirow[t]{7}{*}{ PCV7 CAP } & & 62 & $11.1(8.5-14.2)$ & 9 & $1.6(0.7-3.1)$ & 8 & $1.4(0.6-2.8)$ & 2 & $0.4(0.0-1.3)$ & 13 & $2.3(1.2-4.0)$ \\
\hline & $16-24$ & 0 & 0 & 1 & $0.9(0.0-4.9)$ & 0 & 0 & 0 & 0 & 0 & 0 \\
\hline & $25-44$ & 4 & $2.2(0.6-5.7)$ & 0 & 0 & 1 & $0.6(0.0-3.1)$ & 0 & 0 & 3 & $1.7(0.3-4.9)$ \\
\hline & $45-64$ & 12 & $7.4(3.8-12.9)$ & 3 & $1.8(0.4-5.4)$ & 2 & $1.2(0.0-4.4)$ & 1 & $0.6(0.0-3.4)$ & 1 & $0.6(0.0-3.4)$ \\
\hline & $65-74$ & 15 & $26.7(15.0-44.1)$ & 1 & $1.8(0.0-9.9)$ & 3 & $5.3(1.1-15.6)$ & 1 & $1.8(0.0-9.9)$ & 4 & $7.1(1.9-18.3)$ \\
\hline & $75-84$ & 14 & $39.1(21.4-65.6)$ & 1 & $2.8(0.1-15.6)$ & 1 & $2.8(0.1-15.6)$ & 0 & 0 & 2 & $5.6(0.7-20.2)$ \\
\hline & $>85$ & 17 & $177.1(103.2-283.5)$ & 3 & $31.2(6.4-91.3)$ & 1 & $10.4(0.3-58.0)$ & 0 & 0 & 3 & $31.2(6.4-91.3)$ \\
\hline \multirow[t]{7}{*}{ Additional PCV13 CAP } & & 59 & $10.6(8.0-13.6)$ & 64 & $11.5(8.8-14.6)$ & 45 & $8.1(5.9-10.8)$ & 44 & $7.9(5.7-10.6)$ & 35 & $6.3(4.4-8.7)$ \\
\hline & $16-24$ & 1 & $0.9(0.0-4.9)$ & 1 & $8.7(4.2-16.1)$ & 2 & $1.7(0.2-6.3)$ & 3 & $2.6(0.5-7.7)$ & 3 & $2.6(0.5-7.7)$ \\
\hline & $25-44$ & 9 & $5.0(2.3-9.5)$ & 15 & $8.4(4.7-13.8)$ & 13 & $7.2(3.9-12.4)$ & 4 & $2.2(0.6-5.7)$ & 6 & $3.3(1.2-7.3)$ \\
\hline & $45-64$ & 10 & $6.2(3.0-11.3)$ & 16 & $9.8(5.6-16.0)$ & 11 & $6.7(3.4-12.1)$ & 12 & $7.4(3.8-12.9)$ & 9 & $5.5(2.5-10.5)$ \\
\hline & $65-74$ & 13 & $23.2(12.3-39.6)$ & 11 & $19.6(9.8-35.1)$ & 10 & $17.8(8.6-32.8)$ & 13 & $23.2(12.3-39.6)$ & 7 & $12.5(5.0-25.7)$ \\
\hline & $75-84$ & 16 & $44.7(25.6-72.6)$ & 11 & $30.7(15.3-55.0)$ & 4 & $11.2(3.0-28.6)$ & 7 & $19.6(7.9-40.3)$ & 8 & $22.3(9.6-44.0)$ \\
\hline & $>85$ & 10 & $104.2(50.0-191.6)$ & 10 & $104.2(50.0-191.6)$ & 5 & $52.1(16.9-121.6)$ & 5 & $52.1(16.9-121.6)$ & 2 & $20.8(2.5-75.3)$ \\
\hline \multirow[t]{7}{*}{ Other CAP } & & 24 & $4.3(2.8-6.4)$ & 23 & $4.1(2.6-6.2)$ & 15 & $2.7(1.5-4.4)$ & 12 & $2.1(1.1-3.8)$ & 21 & $3.8(2.3-5.8)$ \\
\hline & $16-24$ & 0 & 0 & 1 & $0.9(0.0-4.9)$ & 0 & 0 & 0 & 0 & 0 & 0 \\
\hline & $25-44$ & 7 & $3.9(1.6-8.0)$ & 3 & $1.7(0.3-4.9)$ & 2 & $1.1(0.1-4.0)$ & 2 & $1.1(0.1-4.0)$ & 4 & $2.2(0.7-5.7)$ \\
\hline & $45-64$ & 7 & $4.3(1.7-8.9)$ & 7 & $4.3(1.7-8.9)$ & 4 & $2.5(0.7-6.3)$ & 4 & $2.5(0.7-6.3)$ & 7 & $4.3(1.7-8.9)$ \\
\hline & $65-74$ & 2 & $3.6(0.4-12.9)$ & 4 & $7.1(1.9-18.3)$ & 4 & $7.1(1.9-18.3)$ & 1 & $1.8(0.0-9.9)$ & 5 & $8.9(2.9-20.8)$ \\
\hline & $75-84$ & 6 & $16.8(6.2-36.5)$ & 6 & $16.8(6.2-36.5)$ & 4 & $11.2(3.0-28.6)$ & 3 & $8.4(1.7-24.5)$ & 3 & $8.4(1.7-24.5)$ \\
\hline & $>85$ & 2 & $20.8(2.5-75.3)$ & 2 & $20.8(2.5-75.3)$ & 1 & $10.4(0.2-58.0)$ & 2 & $20.8(2.5-75.3)$ & 2 & $20.8(2.5-75.3)$ \\
\hline
\end{tabular}


TABLE 3 Linear trends and year to year variation in community-acquired pneumonia (CAP), pneumococcal CAP, CAP due to serotype groups and "untyped" pneumococcal CAP

\begin{tabular}{|c|c|c|c|c|c|c|c|}
\hline & $\begin{array}{c}\text { Year 1, } \\
\text { 2008-2009 }\end{array}$ & $\begin{array}{c}\text { Year 2, } \\
2009-2010\end{array}$ & $\begin{array}{l}\text { Year 3, } \\
2010-11\end{array}$ & $\begin{array}{l}\text { Year 4, } \\
2011-2012\end{array}$ & $\begin{array}{l}\text { Year 5, } \\
2012-13\end{array}$ & $\begin{array}{l}\text { Change in IRR } \\
\text { per year }\end{array}$ & $\begin{array}{l}p \text {-value } \\
\text { for trend }\end{array}$ \\
\hline CAP & 1.00 & $0.9(0.8-1.0)$ & $0.7(0.6-0.8)$ & $1.1(1.0-1.2)$ & $0.7(0.6-0.8)$ & $0.96(0.94-0.99)$ & 0.016 \\
\hline PCV7 CAP & 1.00 & $0.15(0.07-0.29)$ & $0.13(0.06-0.27)$ & $0.03(0.01-0.13)$ & $0.21(0.12-0.38)$ & $0.52(0.43-0.62)$ & $<0.001$ \\
\hline Additional PCV13 CAP & 1.00 & $1.08(0.76-1.54)$ & $0.76(0.52-1.12)$ & $0.75(0.50-1.50)$ & $0.59(0.39-0.90)$ & $0.87(0.80-0.95)$ & 0.002 \\
\hline Other CAP & 1.00 & $0.95(0.54-1.70)$ & $0.62(0.33-1.19)$ & $0.50(0.25-1.00)$ & $0.88(0.49-1.57)$ & $0.91(0.79-1.05)$ & 0.218 \\
\hline
\end{tabular}

Data are presented as incidence rate ratio (95\% confidence interval) unless otherwise stated. PCV7 CAP: CAP due to serotypes included in the 7-valent pneumococcal conjugate vaccine; additional PCV13 CAP: CAP due to additional serotypes included in PCV13; other CAP: CAP due to "other" serotypes. Bold indicates statistically significant.

PCV7 (88\% reduction in CAP due to PCV7 serotypes versus 30\% reduction in CAP incidence due to additional PCV13 serotypes).

\section{Discussion}

To our knowledge, this is the first study to investigate the impact of PCV13 on pneumococcal serotypes implicated in a predominantly non-bacteraemic cohort of pneumococcal CAP adults. The key findings were 1) the incidence of hospitalised pneumococcal pneumonia has been declining over the last 5 years, including the years following PCV13 introduction; 2) the incidence of CAP due to additional PCV13 serotypes declined by $30 \%$ in the 2 years following the introduction of PCV13, when compared to pre-PCV13; and 3) ongoing declines in PCV7-related disease were evident 3-4 years after the introduction of PCV7, the incidence of CAP due to PCV7 serotypes declined by $48 \%$ over the course of the study.

Although studies have previously reported a decline in the incidence of IPD in adults following PCV introduction to infant immunisation schedules [6,7], few have investigated its impact on the incidence of CAP and non-bacteraemic pneumococcal CAP. Analyses from hospital coding databases in the USA have reported a decline in rates of CAP and non-bacteraemic CAP following the introduction of infant PCV7 and PCV13 vaccinations $[16,17]$. A study from the UK on community diagnoses of CAP in children and adolescents $(0-$ 18 years) found a 35\% decline in pneumonia consultations following the introduction of PCV7 between 2006 and 2009 [18]. However, the accuracy of pneumonia diagnoses in coding databases is not verified.

This study observed a $4 \%$ and $16 \%$ decline in CAP and pneumococcal CAP incidence rates per year, respectively. Contemporary national IPD surveillance data from the UK reports a $22 \%$ decline in IPD rates between 2008 and 2010, and between 2012 and 2013, amongst all age groups [19]. A high incidence rate for CAP was observed during 2011-2012, despite a continued decline in pneumococcal CAP in this study. No significant differences in the number of other causative pathogens for CAP were seen during this period in comparison to other years and these numbers remained relatively stable over the 5 years (table S3); however, testing for pathogens other than S. pneumoniae was at the discretion of attending clinical teams and routine testing for atypical pathogens was not conducted. National surveillance data from Public Health England revealed a high number of cases of Mycoplasma pneumoniae in 2011-2012, with similar numbers to 2006 and the highest since 2003 in the UK; hence 2011-2012 coincided with a "Mycoplasma year", which is recognised to peak every $4-7$ years $[20,21]$. National virological surveillance reported relatively lower influenza rates in 2011-2012 in comparison to previous years [22]. Influenza vaccination uptake amongst adults aged $\geqslant 65$ years $(74.1 \%, 72.4 \%, 72.8 \%, 74.0 \%$ and $73.4 \%$ for the years $2008-2009,2009-2010$, 2010-2011, 2011-2012 and 2012-2013, respectively) and "at risk" individuals aged 6 months to <65 years (47.1\%, 51.6\%, 50.4\%, 51.6\% and 51.3\% for the years 2008-2009, 2009-2010, 2010-2011, 2011- 2012 and 2012-013, respectively) remained relatively stable in the UK over the 5 years of the study [15].

In our study, the largest annual reduction in CAP incidence was in adults aged $\geqslant 85$ years. Database analyses from the USA reported the largest decline in CAP hospitalisations in adults $\geqslant 85$ years [16], with similar findings in IPD cohorts elsewhere [7]; although both these studies compared periods before and after PCV7 introduction. Adults at the extremes of age are likely to have the greatest potential for disease reduction from PCVs as rates of disease were highest in these adults prior to PCV introduction. Contemporary national IPD data from the UK showed the largest absolute reduction in IPD rates among adults aged $\geqslant 65$ years, although this decline was attenuated due to a concurrent increase in non-PCV13 


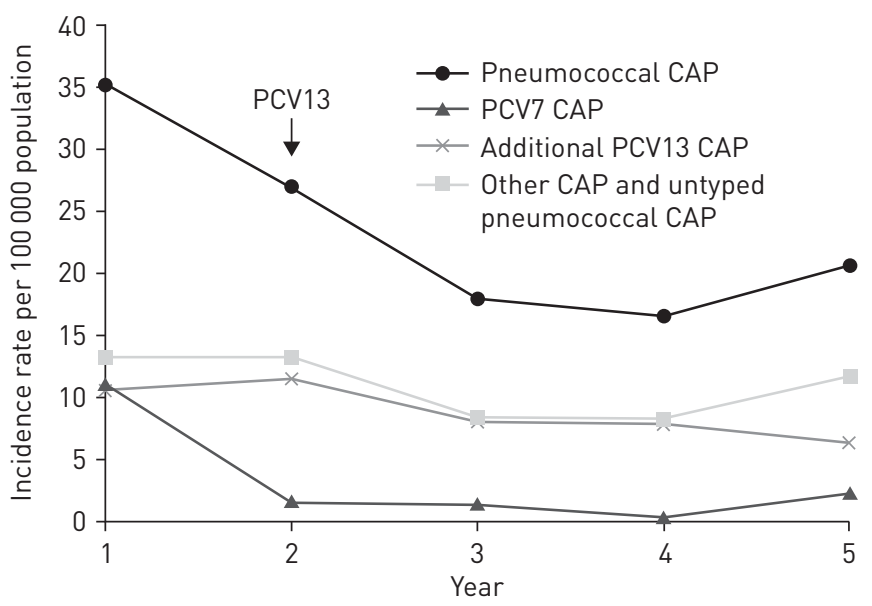

FIGURE 1 Variation in the incidence of adult pneumococcal community-acquired pneumonia (CAP) and CAP by year, according to serotype categories. PCV7 CAP: CAP due to serotypes included in the 7-valent pneumococcal conjugate vaccine; additional PCV13 CAP: CAP due to additional serotypes included in PCV13; other CAP: CAP due to "other" serotypes.

serotypes [19]. The incidence of CAP remains strongly age dependent [23], and the impact of a continually ageing population on CAP incidence in the future requires close observation [24].

Additional PCV13 serotypes accounted for 57\% of all serotyped pneumococcal CAP during the study, with the bulk of disease due to serotypes 1,7F/A, and 19A. The incidence of CAP due to additional PCV13 serotypes was highest during the winter months of 2009-2010, prior to PCV13 introduction in April 2010. Exclusion of this "transitional year" (i.e. 2009-2010) from the analyses, comparing pre- and post-PCV13 periods, is likely to have led to a conservative estimate of the reduction in incidence rates for CAP due to additional PCV13 serotypes.

The incidence of CAP due to PCV7 serotypes declined most markedly between year 1 and year 2 of this study, corresponding to the third-year post-PCV7 introduction, and, thereafter, remained low. A statistically nonsignificant increase in the incidence of CAP due to serotypes $6 \mathrm{~B}$ and $19 \mathrm{~F}$ was observed in the final year of the study; although serotype numbers were small, and, therefore, these specific trends should be interpreted with caution. National IPD surveillance data from the UK found that the majority of vaccine failures among the PCV7 serotypes were due to serotypes $6 \mathrm{~B}$ and $19 \mathrm{~F}$ in the post-PCV7 period [19].

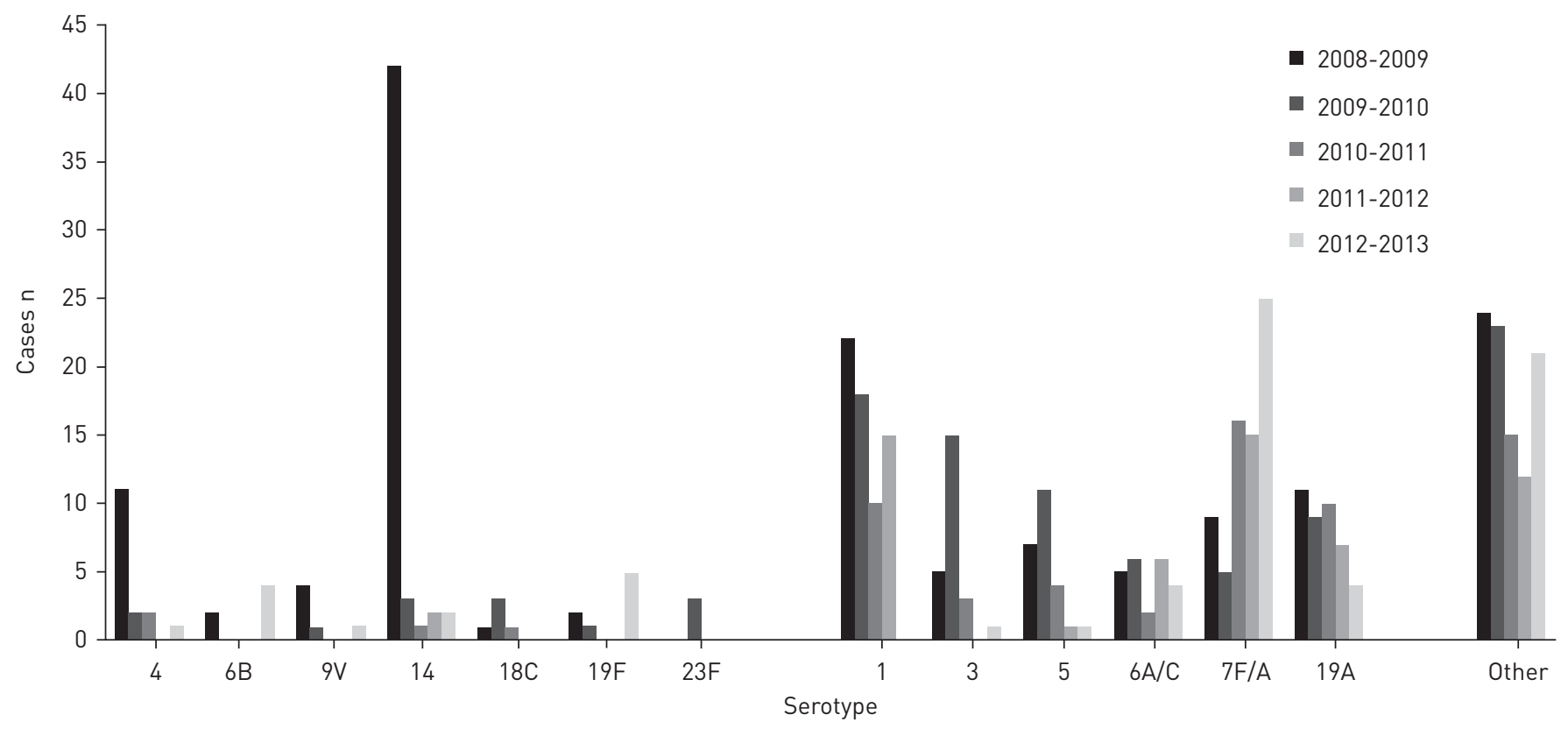

FIGURE 2 Variation in individual serotype numbers by study year among adults with pneumococcal community-acquired pneumonia. 
TABLE 4 Comparison of incidence rates pre-13-valent pneumococcal conjugate vaccine (PCV13) ${ }^{\#}$ and post-PCV13 introduction

\begin{tabular}{|c|c|c|c|c|}
\hline & \multicolumn{2}{|c|}{ Overall incidence $^{+}$} & \multirow[t]{2}{*}{ IRR (95\%CI) } & \multirow[t]{2}{*}{ p-value } \\
\hline & Pre-PCV13 & Post-PCV13 & & \\
\hline CAP & $90.7(83.0-99.0)$ & $76.7(72.6-81.0)$ & $0.85(0.76-0.94)$ & 0.001 \\
\hline Pneumococcal CAP & $35.1(30.4-40.4)$ & $18.3(16.3-20.5)$ & $0.52(0.44-0.62)$ & $<0.001$ \\
\hline PCV7 CAP & $11.1(8.5-14.2)$ & $1.4(0.9-2.1)$ & $0.12(0.08-0.20)$ & $<0.001$ \\
\hline Additional PCV13 CAP & $10.6(8.0-13.6)$ & $7.4(6.2-8.8)$ & $0.70(0.51-0.96)$ & 0.024 \\
\hline Other CAP & $4.3(2.8-6.4)$ & $2.9(2.1-3.8)$ & $0.67(0.41-1.09)$ & 0.105 \\
\hline
\end{tabular}

Data are presented as incidence rates (interquartile range), unless otherwise stated. IRR: incidence rate ratio; CAP: community-acquired pneumonia; PCV7 CAP: CAP due to serotypes included in the PCV7 vaccine; additional PCV13 CAP: CAP due to serotypes included in the PCV13 vaccine; other CAP: CAP due to "other" serotypes. " : years 2008-2009; " : years 2010-2013; ' : per 100000 population. Bold indicates statistically significant.

Vaccine failures related to serotype 19F may be due to resistance to complement deposition and lower sensitivity to opsonophagocytic killing [25]. Inability to effectively clear nasopharyngeal carriage of serotype $19 \mathrm{~F}$ in children may translate into a lack of "herd protection" against serotype 19F amongst adults.

Data from IPD cohorts in the UK and the USA in the years following PCV7 introduction reported serotypes $1,7 \mathrm{~F}$, and 19A as significant replacement serotypes [6,7]. Although the incidence of CAP due to serotypes 1 , 3 and 5 declined during the study, CAP due to serotype 7F/A increased. This finding was in contrast to adult IPD data from the UK and paediatric IPD data from France during the post-PCV13 period [19, 26]. Although the Bio-Plex assay used in this study was unable to differentiate between serotypes 7F and 7A, it is likely that the majority of implicated serotypes were 7F, as previous studies of both IPD and non-invasive pneumococcal disease have shown that serotype $7 \mathrm{~A}$ is rarely implicated $[6,7,27]$, Differences in serotypes implicated in contemporary cohorts of IPD and non-bacteraemic pneumococcal disease have been demonstrated previously [28]. Further, unvaccinated adults are likely to have a residual burden of pneumococcal disease due to serotypes included in PCVs, and herd protection effects are likely to manifest slower, in comparison to the vaccinated paediatric population $[6,7]$.

\section{Strengths and weaknesses}

A major strength of this large prospective cohort study is the robust case ascertainment of radiologically confirmed CAP, drawn from a relative stable population over a 5-year period. Together the two teaching hospitals in this study serve the catchment area of Greater Nottingham comprising 557800 adults [14]. Comprehensive inpatient services have been provided for this population by the same two hospitals for more than two decades. Relative to other regions of the UK, Nottinghamshire ranks as "average" with regard to indices of health, socioeconomic deprivation and ethnic diversity $[29,30]$. The base population is, therefore, a representative reflection of the UK.

The main limitation in our study is that reliable inferences cannot be drawn regarding trends in CAP rates due serotypes not included in PCV13. Around 25-38\% of the pneumococcal cohort was "untyped" over the 5 -year period of the study (table S4); this mainly represents the proportion of cases that were BinaxNOW positive but Bio-Plex negative. Although the incidence rates of "untyped" pneumococcal CAP remained relatively stable over the study period (5.7-8.7 per 100000 adults), national IPD data demonstrate a $22 \%$ and $31 \%$ increase in non-PCV13 serotypes in adults aged $45-64$ years and $\geqslant 65$ years, respectively, between 2008-2010 and 2012-2013 (unpublished data from Public Health England). Extrapolating the proportion of non-PCV13 serotypes in bacteraemic adults (28.7\%) to the untyped pneumococcal cohort in this study, showed a relatively stable number of adults with non-PCV13 serotypes over the 5 years (table S5).

The incidence of CAP in this study was lower in comparison to a previous study in the UK based on hospital coding data from the pre-PCV era [2]. This difference is partly explained by inaccuracies in the coding of CAP within the UK Hospital Episode Statistics database; various studies indicate that in about $50 \%$ of coded pneumonias, there is no evidence of pneumonia on the chest radiograph [31,32]. Some of the difference may also be due to a true reduction in the overall incidence of CAP in recent years compared to the pre-PCV period $[16,33]$.

The severity of CAP in hospitalised adults remained stable during the 5-year period of our study, reflecting a consistent threshold for hospital admission for pneumonia, in line with national guidelines over this period. As this study did not encompass patients treated for pneumonia in the community, it is theoretically possible that the incidence of overall pneumonia (community and hospital treated) has remained stable over the 
study period, but that the overall severity of pneumonia has reduced with a resultant decrease in the rate of hospitalisations. Further community based studies are needed to explore this potential.

The combined sensitivity of the BinaxNOW (74\%) and Bio-Plex tests (79\%) utilised in this study is considerably higher than conventional cultural techniques, especially following the administration of antibiotics $[10,11,13]$. Nevertheless, a proportion of pneumococcal disease may have been missed leading to an underestimate of the true incidence of disease. This is unlikely to affect the analysis of trends in incidence rates of pneumonia due to PCV serotypes. The specificity of the BinaxNOW test and Bio-Plex assay is $\geqslant 97 \%[10,11]$.

In conclusion, in a population with high infant PCV coverage, incidence rates of adult pneumococcal pneumonia have declined over the last 5 years. The introduction of PCV13 infant vaccination was followed by reductions in CAP due to PCV13 serotypes indicating early herd protection effects on adult bacteraemic and non-bacteraemic disease that may continue to accrue, as observed following PCV7 introduction. These data have implications for pneumococcal vaccination policies in adults as the residual burden of PCV13 serotype disease in adults following implementation of childhood immunisation may be too low for an adult programme to be cost-effective.

\section{Acknowledgements}

The authors would like to thank Tim Harrison from for his firm support and Sally-Ann Nguyen, Christine More and Seyi Eletu for processing the urine samples using the Bio-Plex assay (all Respiratory and Vaccine Preventable Bacteria Reference Unit, Public Health England, Colindale, UK); Robert Cave, Andrew Shelton, Adrian Patrick, Michelle Stannard and Joanne Palfreyman (Microbiology Dept, Nottingham University Hospitals, Nottingham, UK) for processing the urine specimens using the BinaxNOW assay; the clinicians and staff of Nottingham University Hospitals NHS Trust (Nottingham, UK); and Gemma Thompson, Emily Jarvis, Melanie Caine and Gaynor Bates (Nottingham University Hospitals NHS Trusts, Nottingham, UK) for assisting with patient recruitment. The authors acknowledge the support of Alere in providing BinaxNOW test kits towards the conduct of this study.

\section{References}

1 Strehlow MC, Emond SD, Shapiro NI, et al. National study of emergency department visits for sepsis, 1992 to 2001. Ann Emerg Med 2006; 48: 326-331.e3.

2 Trotter CL, Stuart JM, George R, et al. Increasing hospital admissions for pneumonia, England. Emerg Infect Dis 2008; 14: 727-733.

3 Lim WS, Macfarlane JT, Boswell TC, et al. Study of community acquired pneumonia aetiology (SCAPA) in adults admitted to hospital: implications for management guidelines. Thorax 2001; 56: 296-301.

4 van der Eerden MM, Vlaspolder F, de Graaff CS, et al. Value of intensive diagnostic microbiological investigation in low- and high-risk patients with community-acquired pneumonia. Eur J Clin Microbiol Infect Dis 2005; 24: 241-249.

5 Public Health England. Pneumococcal disease: guidance, data and analysis. www.gov.uk/government/collections/ pneumococcal-disease-guidance-data-and-analysis Date last accessed: September 2013.

6 Miller E, Andrews NJ, Waight PA, et al. Herd immunity and serotype replacement 4 years after seven-valent pneumococcal conjugate vaccination in England and Wales: an observational cohort study. Lancet Infect Dis 2011; 11: 760-768.

7 Pilishvili T, Lexau C, Farley MM, et al. Sustained reductions in invasive pneumococcal disease in the era of conjugate vaccine. J Infect Dis 2010; 201: 32-41.

8 Sherwin RL, Gray S, Alexander R, et al. Distribution of 13-valent pneumococcal conjugate vaccine Streptococcus pneumoniae serotypes in US adults aged $\geqslant 50$ years with community-acquired pneumonia. J Infect Dis 2013; 208 : $1813-1820$

9 Rozenbaum $\mathrm{MH}$, van Hoek AJ, Fleming D, et al. Vaccination of risk groups in England using the 13 valent pneumococcal conjugate vaccine: economic analysis. BMJ 2012; 345: e6879.

10 Sinclair A, Xie X, Teltscher M, et al. Systematic review and meta-analysis of a urine-based pneumococcal antigen test for diagnosis of community-acquired pneumonia caused by Streptococcus pneumoniae. J Clin Microbiol 2013; 51: 2303-2310.

11 Sheppard CL, Harrison TG, Smith MD, et al. Development of a sensitive, multiplexed immunoassay using xMAP beads for detection of serotype-specific Streptococcus pneumoniae antigen in urine samples. J Med Microbiol 2011; 60: 49-55.

12 Ewig S, Schlochtermeier M, Goke N, et al. Applying sputum as a diagnostic tool in pneumonia: limited yield, minimal impact on treatment decisions. Chest 2002; 121: 1486-1492.

13 Musher DM, Montoya R, Wanahita A. Diagnostic value of microscopic examination of Gram-stained sputum and sputum cultures in patients with bacteremic pneumococcal pneumonia. Clin Infect Dis 2004; 39: 165-169.

14 Nottingham City Council. Nottingham Insight: Census 2011. 2011. www.nottinghaminsight.org.uk/insight/ key-datasets/census-2011.aspx. Date last accessed: May 2014.

15 Public Health England. Vaccine uptake guidance and the latest coverage data. 2014. www.gov.uk/government/ collections/vaccine-uptake Date last accessed: June 2014.

16 Griffin MR, Zhu Y, Moore MR, et al. U.S. hospitalizations for pneumonia after a decade of pneumococcal vaccination. N Engl J Med 2013; 369: 155-163.

17 Simonsen L, Taylor RJ, Young-Xu Y, et al. Impact of pneumococcal conjugate vaccination of infants on pneumonia and influenza hospitalization and mortality in all age groups in the United States. MBio 2011; 2: e00309-e00310. 
18 El Turki A, Hsia Y, Saxena S, et al. Impact of 7-valent pneumococcal conjugate vaccine (PVC-7) on the incidence and treatment of pneumonia diagnosed in primary care in children and adolescents in UK. Arch Dis Child 2011; 96: Suppl. 1, A5.

19 Kaye P, Malkani R, Slack M, et al. Real-time surveillance to evaluate the impact of the pneumococcal conjugate vaccine (PCV) in England and Wales. Pneumonia 2014; 3: 174.

20 Public Health England. Laboratory reports to HPA Colindale of infections due to Mycoplasma pneumoniae England and Wales by date of report 1991-2013 (4 weekly). Available from: www.gov.uk/mycoplasma Date last accessed: June 2014. Date last updated.

21 Chalker V, Stocki T, Litt D, et al. Increased detection of Mycoplasma pneumoniae infection in children in England and Wales, October 2011 to January 2012. Euro Surveill 2012; 17: pii 20081.

22 Public Health England. Health Protection-Collection. Seasonal influenza: guidance, data and analysis. www.gov.uk/ government/collections/seasonal-influenza-guidance-data-and-analysis\#epidemiology Date last accessed: November. Date last updated July 14, 2014.

23 Ewig S, Birkner N, Strauss R, et al. New perspectives on community-acquired pneumonia in 388406 patients. Results from a nationwide mandatory performance measurement programme in healthcare quality. Thorax 2009; 64: 1062-1069.

24 Wroe PC, Finkelstein JA, Ray GT, et al. Aging population and future burden of pneumococcal pneumonia in the United States. J Infect Dis 2012; 205: 1589-1592.

25 Melin M, Jarva H, Siira L, et al. Streptococcus pneumoniae capsular serotype 19F is more resistant to C3 deposition and less sensitive to opsonophagocytosis than serotype 6B. Infect Immun 2009; 77: 676-684.

26 Angoulvant F, Levy C, Grimprel E, et al. Early impact of 13-Valent pneumococcal conjugate vaccine on community-acquired pneumonia in children. Clin Infect Dis 2014; 58: 918-924.

27 Okade H, Funatsu T, Eto M, et al. Impact of the pneumococcal conjugate vaccine on serotype distribution and susceptibility trends of pediatric non-invasive Streptococcus pneumoniae isolates in Tokai, Japan over a 5-year period. J Infect Chemother 2014; 20: 423-428.

28 Bewick T, Sheppard C, Greenwood S, et al. Serotype prevalence in adults hospitalised with pneumococcal non-invasive community-acquired pneumonia. Thorax 2012; 67: 540-545.

29 Public Health England. Longer lives. http://healthierlives.phe.org.uk/topic/mortality Date last accessed: July 2014.

30 Office for National Statistics. 2011 Census-Ethnicity. www.ons.gov.uk/ons/interactive/census-map-2-1---ethnicity/ index.html Date last accessed: July 2014.

31 Price L, Anwar M, Srikanthan K, et al. P244 accuracy of admission diagnosis of pneumonia. Thorax 2013; 68: Suppl. 3, A187.

32 Pink KL, Mitchell I, Davies HE. Clinical observation in acute and chronic lung infection: P17 the accuracy of a diagnosis of pneumonia in a UK teaching hospital. Thorax 2012; 67: Suppl. 2, A71.

33 Simonsen L, Taylor RJ, Schuck-Paim C, et al. Effect of 13-valent pneumococcal conjugate vaccine on admissions to hospital 2 years after its introduction in the USA: a time series analysis. Lancet Respir Med 2014; 2: 387-394. 